\title{
Glucagon Clearance is Preserved in Type 2 Diabetes
}

Magnus F.G. Grøndahl*1, Asger Lund*1, Jonatan I. Bagger ${ }^{1}$, Tonny S. Petersen ${ }^{2,5}$, Nicolai J. Wewer Albrechtsen ${ }^{3,5}$, Jens J. Holst ${ }^{4,5}$, Tina Vilsbøll ${ }^{1,2,6}$, Mikkel B. Christensen ${ }^{1,2,7}$, Filip K.

$$
\text { Knop }^{1,2,3,6}
$$

${ }^{1}$ Center for Clinical Metabolic Research, Gentofte Hospital, University of Copenhagen, Hellerup, Denmark, ${ }^{2}$ Department of Clinical Medicine, Faculty of Health and Medical Sciences, University of Copenhagen, Copenhagen, Denmark, ${ }^{3}$ Department of Clinical Biochemistry, Rigshospitalet, University of Copenhagen, Copenhagen, Denmark, ${ }^{4}$ Novo Nordisk Foundation Center for Basic Metabolic Research, Faculty of Health and Medical Sciences, University of Copenhagen, Copenhagen, Denmark, ${ }^{5}$ Department of Biomedical Sciences, Faculty of Health and Medical Sciences, University of Copenhagen, Copenhagen, Denmark, ${ }^{6}$ Steno Diabetes Center Copenhagen, Gentofte, Denmark; ${ }^{7}$ Department of Clinical Pharmacology, Bispebjerg University Hospital, Copenhagen, Denmark

*These authors contributed equally to this paper.

Correspondence to: Filip K. Knop, MD PhD, Center for Clinical Metabolic Research, Gentofte Hospital, University of Copenhagen, Gentofte Hospitalsvej 7, $3^{\text {rd }}$ floor, DK-2900 Hellerup, Denmark; Phone: +45 38674266; fax: +45 38672689; e-mail: filip.krag.knop.01@ regionh.dk

\section{Supplemental Material}

Supplemental Tables: 1; Supplemental Figures: 4 
Table S1. Population pharmacokinetic parameters

\begin{tabular}{|lll|}
\hline Parameter & Population estimate & Between-subject variability \\
\hline $\mathrm{Vd}(\mathrm{L})$ & $8.04(8.4)$ & $0.244(14)$ \\
$\mathrm{B}_{\text {body weight, } \mathrm{Vd}}$ & $1.1(20)$ & \\
$\mathrm{B}_{\text {glucose, } \mathrm{Vd}}$ & $0.403(35)$ & \\
$\mathrm{Cl}\left(\mathrm{L}^{*} \mathrm{~min}^{-1}\right)$ & $1.27(5.2)$ & $0.152(14)$ \\
$\mathrm{B}_{\text {body weight, Cl }}$ & $0.797(17)$ & \\
$\mathrm{B}_{\text {glucose, } \mathrm{Cl}}$ & $0.441(19)$ & \\
$\mathrm{EG}\left(\mathrm{pmol}{ }^{*} \mathrm{~min}^{-1}\right)$ & $10.3(11)$ & \\
$\mathrm{B}_{\text {HOMA-R, EG }}$ & $1.02(23)$ & \\
Additive error $\left(\mathrm{pmol} \mathrm{L}^{-1}\right)$ & $1.63(9.4)$ & \\
Proportional error & $0.0687(9.3)$ & \\
\hline
\end{tabular}

Coefficients regarding Figure 3. Data is presented as mean (relative standard error (\%)). B body weight, Vd, bodyweight influential parameter on $\mathrm{V}_{\mathrm{d}}$. $\mathrm{B}_{\text {body weight, } \mathrm{Cl}}$, bodyweight influential parameter on $\mathrm{Cl}$. $\mathrm{B}_{\text {glucose, }} \mathrm{Cl}$, plasma glucose influential

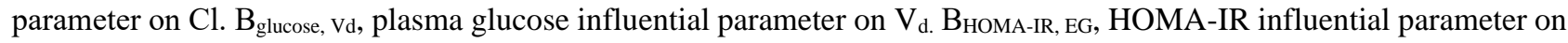
EG. $\mathrm{Cl}$, clearance for a $70 \mathrm{~kg}$ person. EG, endogenous glucagon secretion. HOMA-IR, homeostasis model assessment insulin resistance. RSE, relative standard error. Vd, population volume of distribution for a $70 \mathrm{~kg}$ person. 


\section{Supplemental figure S1.}

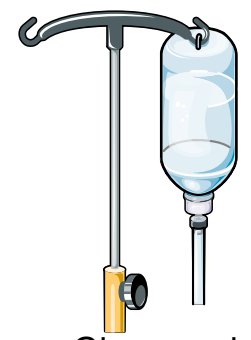

Glucagon infusion rate
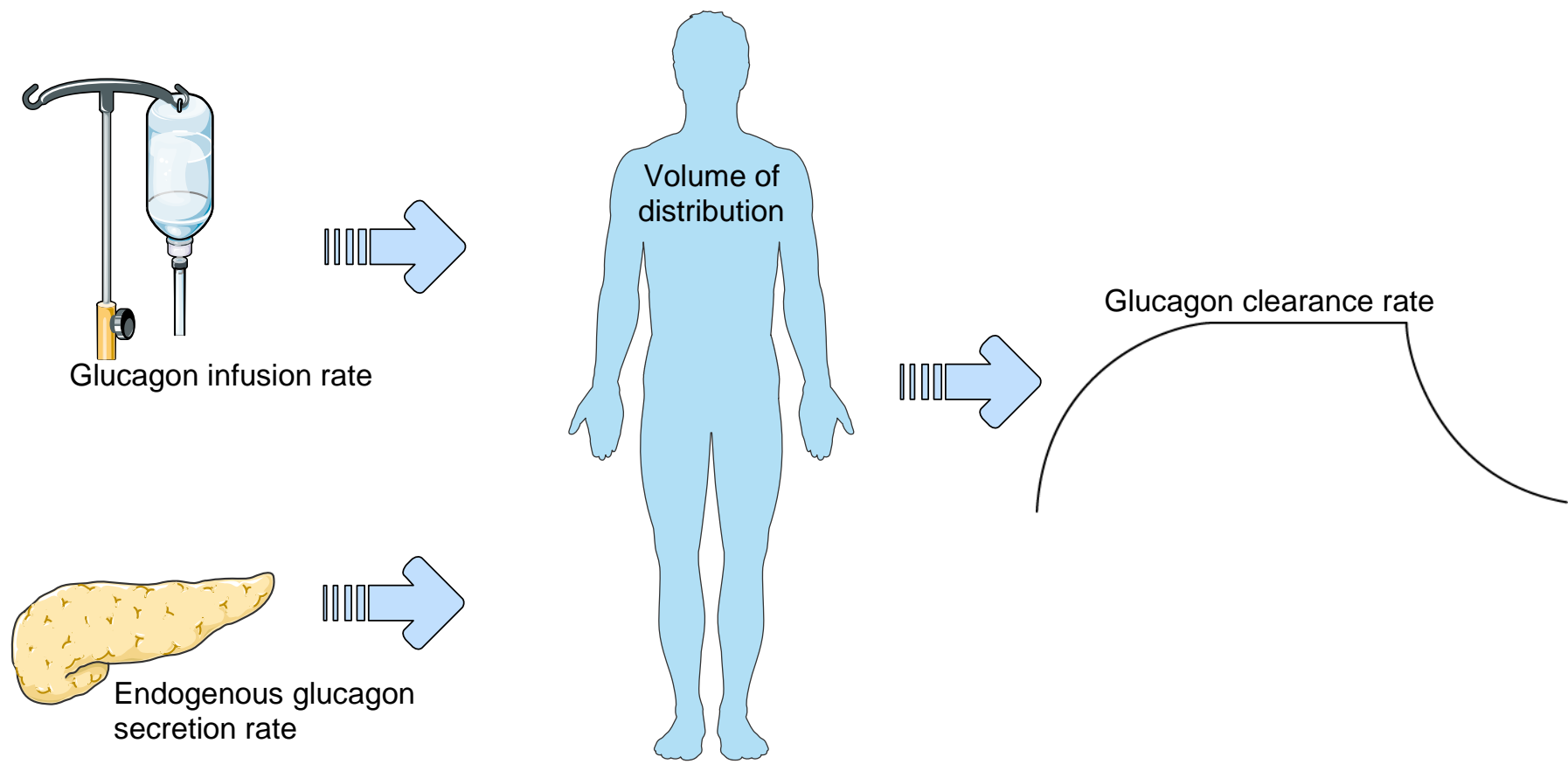

Simplistic overview of the pharmacokinetic model, assuming one-compartment distribution of glucagon. 


\section{Supplemental figure S2}

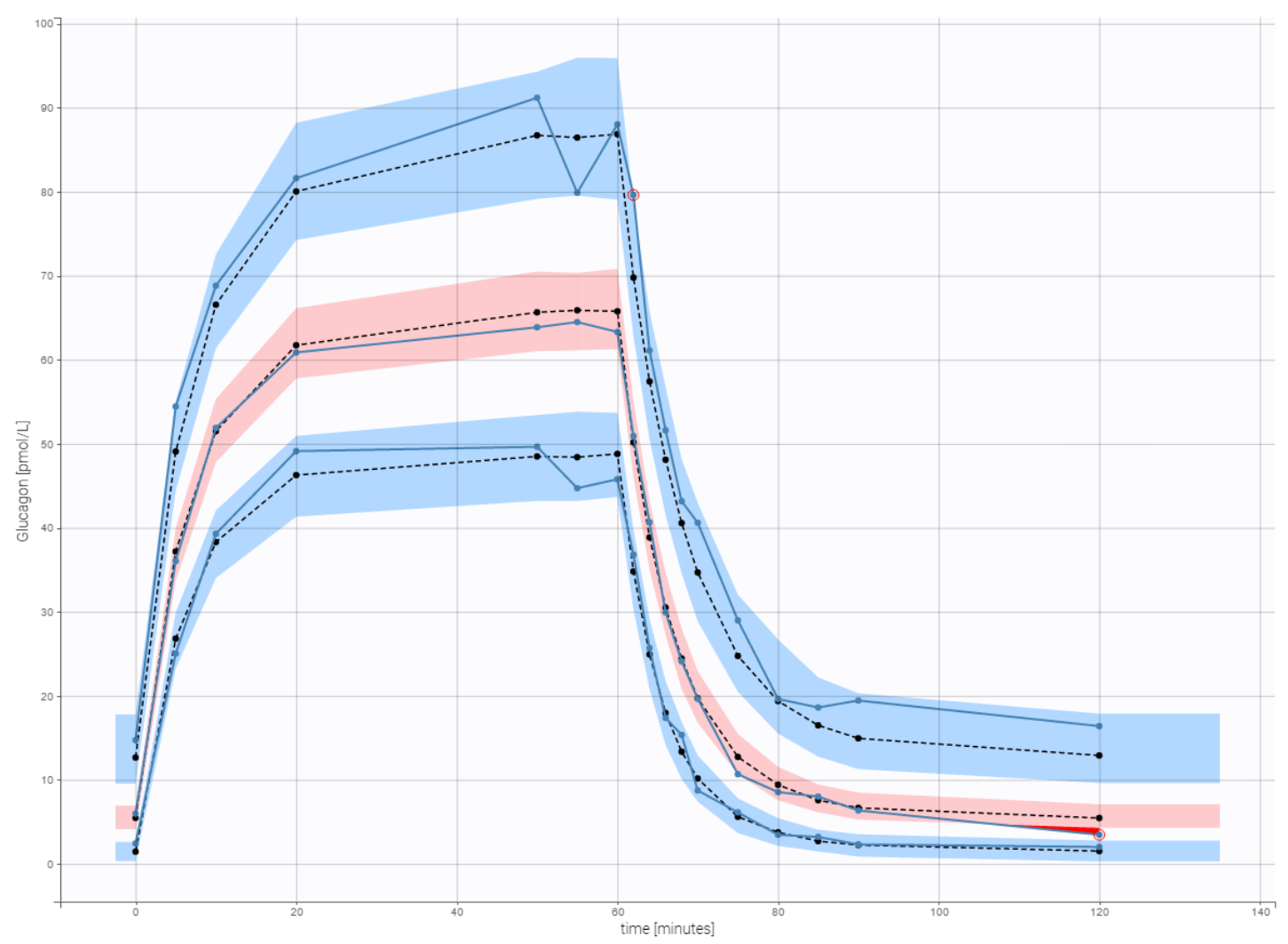

Comparison of observed and predicted glucagon pharmacokinetics. Visual predictive check for glucagon concentrations vs time in minutes. Glucagon infusion was initiated at time $0 \mathrm{~min}$. The lines depict the $5^{\text {th }}, 50^{\text {th }}$ and $95^{\text {th }}$ percentiles of observed (blue lines) and predicted (dashed black lines) glucagon concentration vs time. The colored areas represent the 95\% confidence interval around the simulated percentiles (i.e. dashed black lines) of the $5^{\text {th }}$ and $95^{\text {th }}$ percentiles (blue areas) and $50^{\text {th }}$ percentile (pink area) of the median predicted glucagon concentration vs. time. 
Supplemental figure S3
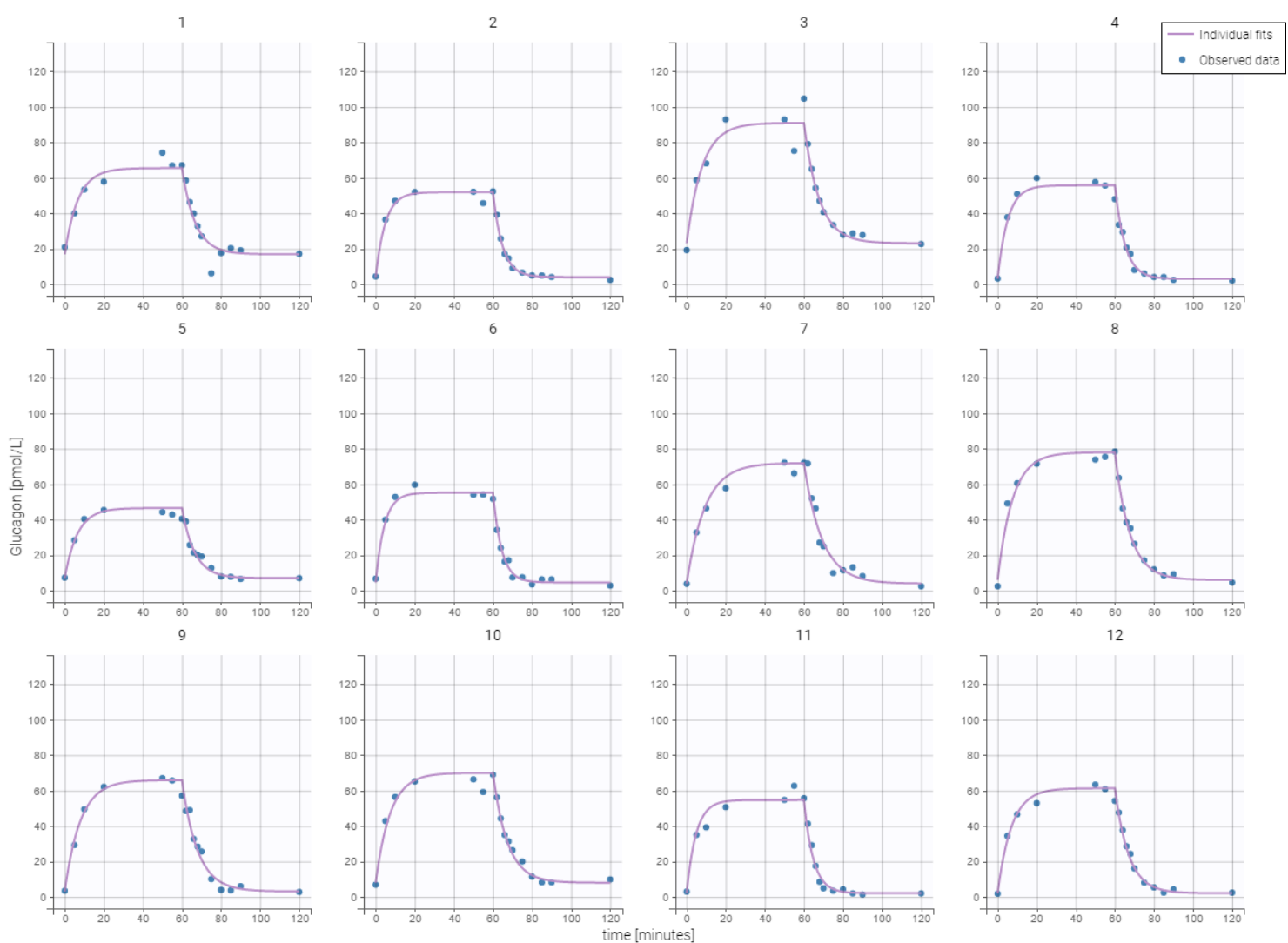

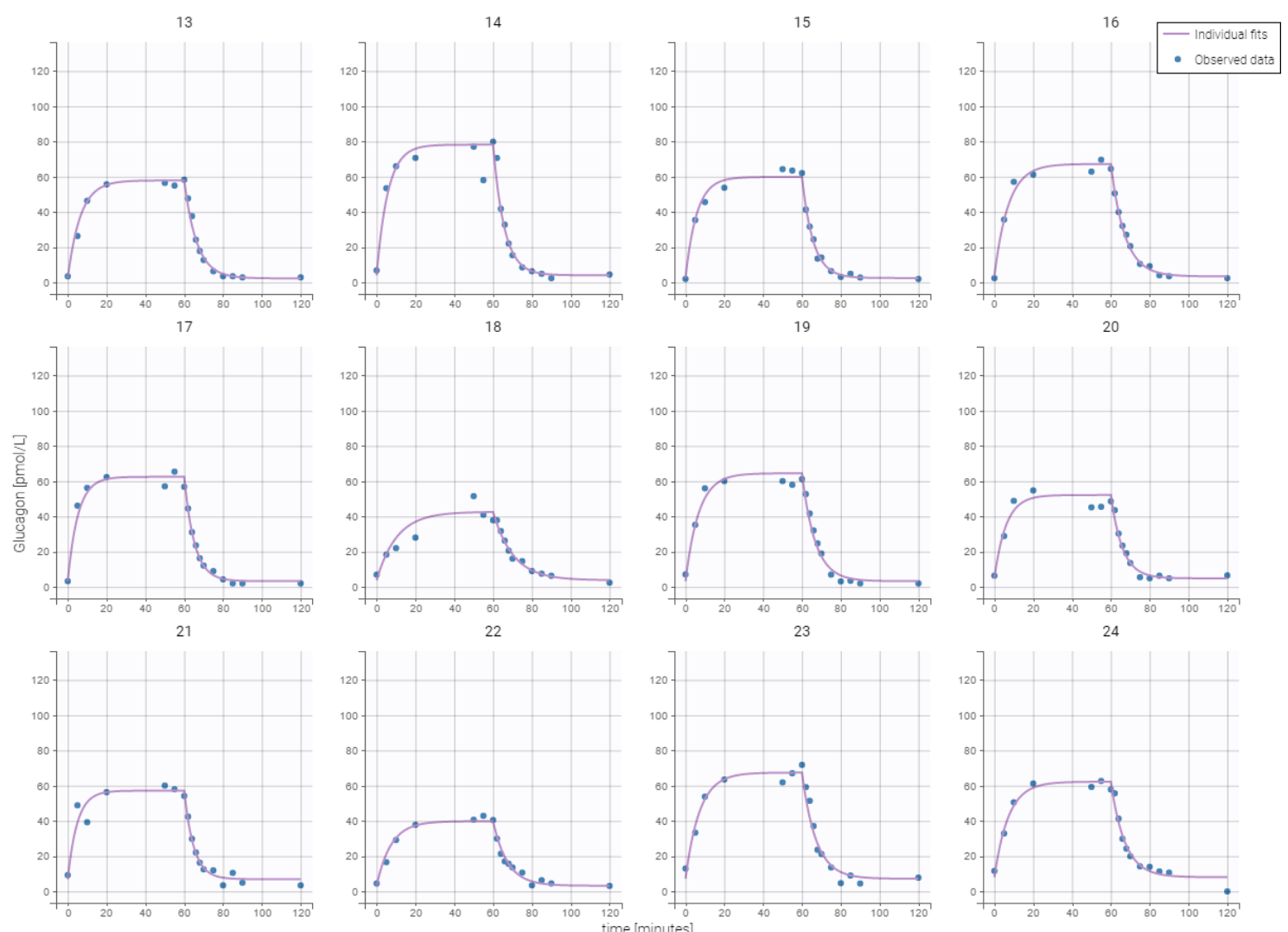

25
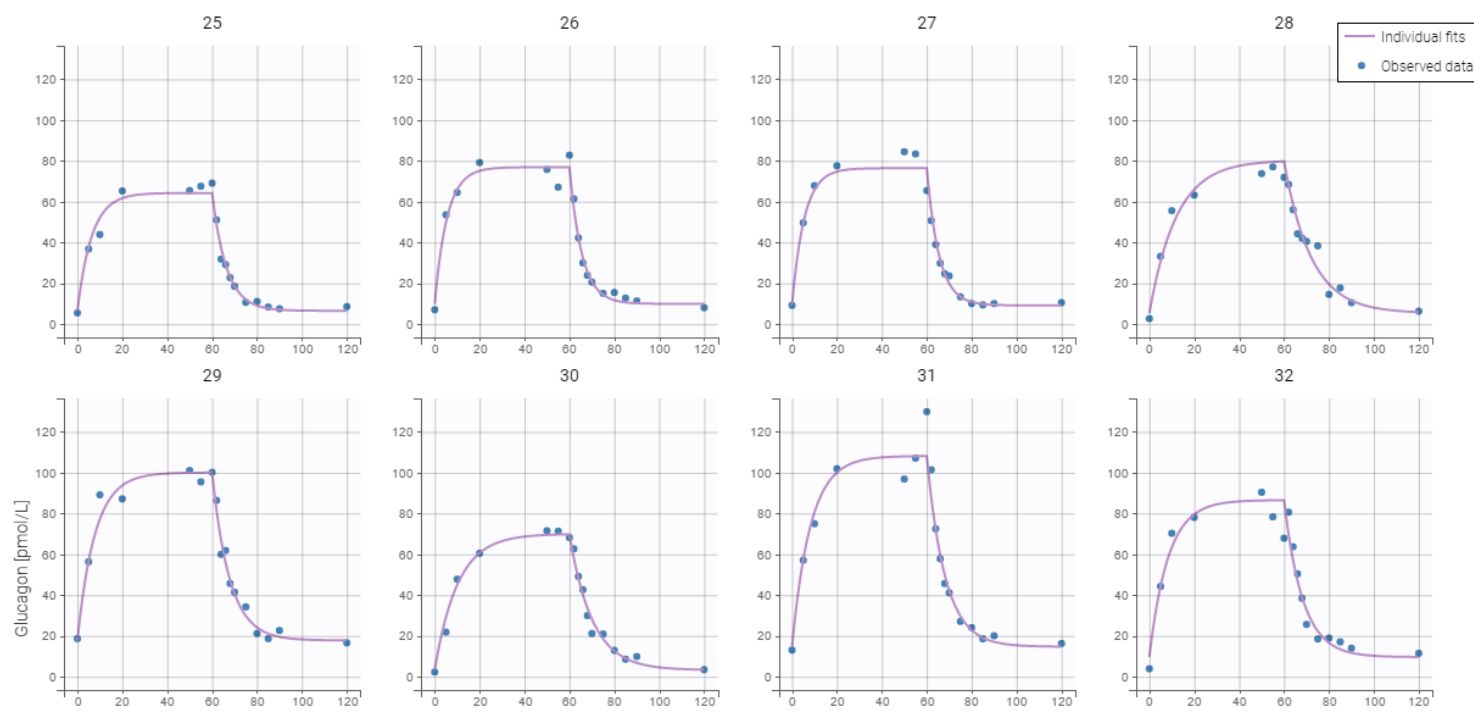

Figure S3. Individual visual predictive checks for model fit. Each subfigure represent an individual fit derived from the pharmakokinetic model (purple line) compared to the factual measured glucagon values in each of the 32 subjects (blue dots). 


\section{Supplemental figure S4}

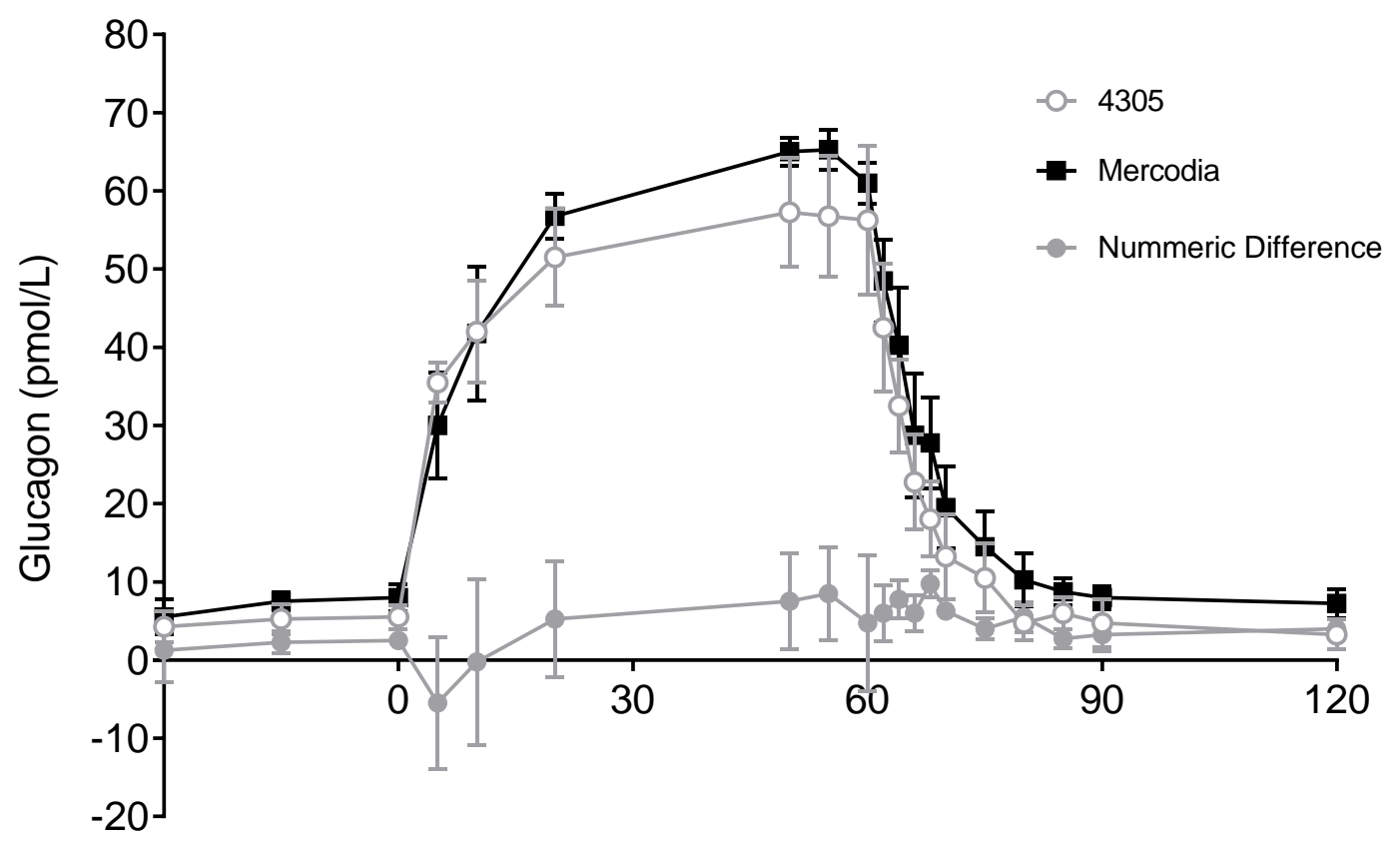

Time $(\min )$

Figure S4: Comparison of analyses made with RIA (4305, grey curve, open symbols) and ELISA (Mercodia, black curve, solid symbols) kits. 